\title{
Construction of ceRNA networks reveals differences between distal and proximal colon cancers
}

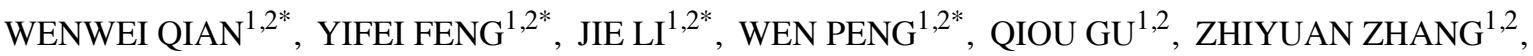 \\ DONGJIAN JI ${ }^{1,2}$, QINGYUAN WANG ${ }^{1,2}$, DONGSHENG ZHANG ${ }^{2}$ and YUEMING SUN ${ }^{2}$ \\ ${ }^{1}$ The First School of Clinical Medicine, Nanjing Medical University; ${ }^{2}$ Department of General Surgery, \\ The First Affiliated Hospital of Nanjing Medical University, Nanjing, Jiangsu 210029, P.R. China
}

Received October 26, 2018; Accepted March 19, 2019

DOI: $10.3892 /$ or.2019.7083

\begin{abstract}
Although colon cancer is often referred to as a homogeneous entity, an increasing number of studies have revealed that colon cancer can be divided according to the anatomic site of the cancer. However, few studies have reported the difference between distal and proximal colon cancer with regard to molecular mechanism, and especially non-coding RNA molecules. In the present study, the data of 186 colon tumour tissues and 17 adjacent non-tumour colon tissues in the left colon and 229 colon tumour tissues and 21 adjacent non-tumour colon tissues in the right colon were obtained from The Cancer Genome Atlas (TCGA). A total of 879 lncRNAs, 165 miRNAs and 2,028 mRNAs were identified as left-specific RNAs [ $\log _{2}$ (fold change) $>2$, FDR $\left.<0.01\right]$. There were 916 lncRNAs, 227 miRNAs and 2,069 mRNAs identified in right colon cancer. The Kyoto Encyclopaedia of Genes and Genomes (KEGG) pathways were analysed for 2,028 mRNAs from left colon cancer and 2,069 mRNAs from right colon cancer. After removing the elements of the intersection from side-specific lncRNAs of the left and right, we identified specific lncRNAs included exclusively in left or right colon cancer, including 277 lncRNAs in left colon cancer and 314 lncRNAs in right colon cancer. Among these lncRNAs, 20 lncRNAs from the left and 25 lncRNAs from the right
\end{abstract}

Correspondence to: Dr Yueming Sun, Department of General Surgery, The First Affiliated Hospital of Nanjing Medical University, 300 Guangzhou Road, Nanjing, Jiangsu 210029, P.R. China

E-mail: jssym@vip.sina.com

*Contributed equally

Abbreviations: CRC, colorectal cancer; lncRNAs, long noncoding RNAs; DElncRNAs, differentially expressed lncRNAs; RIDElncRNAs, DElncRNAs after removing the elements of the intersection of the left and right colon cancer; miRNAs, microRNAs; KEGG, Kyoto Encyclopaedia of Genes and Genomes; ceRNAs, competing endogenous RNAs

Key words: distal and proximal colon cancer, lncRNA, ceRNA network, bioinformatics analysis were revealed to be associated with overall survival. Then, ceRNA networks were constructed. There were 18 lncRNAs, 22 miRNAs and 57 mRNAs included in the left colon cancer ceRNA network and 21 lncRNAs, 27 miRNAs and 55 mRNAs included in the right ceRNA network. In total, 15 lncRNAs were revealed to be significantly related to clinical features, two of which were ascertained by testing the mRNA expression of tissues. In conclusion, our research aimed to detect the difference between colon cancer in the left and the right colon and to assist in the identification of new potential biomarkers to be used for diagnostic and prognostic purposes.

\section{Introduction}

Despite the increased understanding of its pathogenic risk and the development of progressive therapeutic strategies, colorectal cancer (CRC) is the third most commonly diagnosed cancer in men and the second most commonly diagnosed cancer in women worldwide (1).

Colon cancer is often referred to as a homogeneous entity, which should be treated accordingly. However, recent advances on CRC that have identified more subgroups of colon cancer may challenge this concept. These studies revealed that colon cancer can be divided into two subgroups (proximal and distal to the splenic flexure), with specific molecular, clinical and pathological characteristics (2-4). Biological differences between the left and right colon may partly explain the significant heterogeneity of the two sides. The difference of embryonic derivation should take into account that proximal and distal colon originate from the midgut and the hindgut, respectively, which may be the initiating factor (5). Additionally, the differential bacterial flora from the left and right colon may contribute to the heterogeneity (6). Thus, the location of the tumour may be an important factor that is worth taking into consideration. However, little is known about the molecular mechanism, especially with regard to non-coding RNA molecules. To further detect the potentially location-related mechanisms, cancer-specific RNAs from left and right colon cancer were identified and a competing endogenous RNA (ceRNA) network was established based on 3 types of RNAs, including long non-coding RNAs (lncRNAs), microRNAs (miRNAs) and mRNAs, which were differentially expressed in the two sides. 
Non-coding RNAs (ncRNAs), which play crucial biological roles (7), can be divided into small ncRNAs ( $<200 \mathrm{bp})$ and long ncRNAs ( $>200 \mathrm{bp}$ ) based on the number of base pairs. Numerous studies have revealed that lncRNAs play important roles in the process of tumourigenesis (8-10). The competing endogenous RNA (ceRNA) hypothesis presented by Salmena et al in 2011 indicated a regulatory RNA network (11). All types of RNA transcripts, including mRNAs, lncRNAs and pseudogene transcripts in the network, could communicate with each other and compete for the binding of miRNA response elements (MREs). This competition exerts a crucial role in tumourigenesis by affecting the expression levels of various RNAs through MREs.

Several studies on the differences between the proximal and distal colon in pathway activation and their clinical implications have been reported $(12,13)$. However, there is still a lack of large sample size studies and cancer-specific lncRNA biomarkers concerning the differences of the colon sides, and almost none of the studies focused on the potential ceRNA network. To detect the relationship between RNAs of these two sides, data was downloaded from The Cancer Genome Atlas (TCGA) (http://cancergenome.nih.gov), which contains mRNA, miRNA and lncRNA data of 186 samples of tumour tissues and 17 adjacent non-tumour colon tissues in the left colon and 229 samples of tumour tissues and 21 adjacent non-tumour colon tissue in the right colon. To the best of our knowledge, our study is the first to use a large-scale sequencing database to explore the side-specific lncRNA expression profiles and ceRNA co-expression network in the proximal and distal colon. The present study may further our insight into the potentially location-related mechanisms and help clarify the functions of lncRNAs in colon cancer.

\section{Materials and methods}

Patients and samples. RNA expression and clinical data were downloaded, including sex, TNM stage, survival information, from TCGA database. The criteria of exclusion were set as follows: i) Histologic diagnosis was not colon cancer; ii) tissue samples without enough data for analysis; iii) patients who suffered other malignancies; and iv) patients who had received preoperative chemotherapy. A total of 186 colon tumour tissues and 17 adjacent non-tumour colon tissues in the left colon and 229 colon tumour tissues and 21 adjacent non-tumour colon tissues in the right colon were collected in the present study. Our study fully abided by the publication guidelines of TCGA, and thus the approval of an Ethics Committee was not required.

A total of 116 paired colon cancer tissue samples (58 pairs from both sides) were surgically obtained between June 2005 and June 2018 at The First Affiliated Hospital of Nanjing Medical University (Jiangsu, China). Our study was approved by the Research Ethics Committee of Nanjing Medical University, and informed consent was obtained from all patients. All the tissues were frozen in liquid nitrogen immediately after surgical excision and stored at $-80^{\circ} \mathrm{C}$. The TNM stage was classified on the basis of the National Comprehensive Cancer Network (NCCN) guidelines. Patients who received any preoperative treatments were not included in the present study.
RNA sequence data sets and computational analysis. The colon cancer (COAD) RNA expression prolife data (level 3) was downloaded from TCGA database (September 2017) (http://cancergenome.nih.gov). We obtained the normalized count data of RNA sequencing, including lncRNA and mRNA expression profiles. Level 3 miRNAseq data was obtained from TCGA by an Illumina HiSeq 2000 miRNA sequencing (miRNAseq) platform (Illumina, Inc., Hayward, CA, USA). First, the tumour samples were divided into 2 groups (left and right colon cancer). Then, we compared the differentially expressed lncRNAs (DElncRNA), mRNAs (DEmRNA) and miRNAs (DEmiRNA) between tumour tissues and adjacent non-tumour tissues using the Empirical Analysis of Digital Gene Expression Data package in R (edgeR, $\mathrm{R}$ version 3.4.1, http://www.bioconductor.org/packages/) [absolute $\log _{2}$ (fold change) $>2.0$, FDR $<0.01]$ in these 2 groups. In the next step, the intersecting lncRNAs were identified in the aforementioned 2 groups. After removing the elements of the intersection from DElncRNAs (RIDElncRNAs) of the left and right side, the DElncRNAs included exclusively in left or right colon cancer were obtained. Fig. 1 displays the bioinformatics analysis process.

Construction of the ceRNA network. According to the relationship among lncRNAs, miRNAs and mRNAs and the theory that IncRNAs can regulate miRNAs by binding them and further regulate mRNAs, a ceRNA network was constructed. miRcode (http://www.mircode.org/) was used to predict the differentially expressed miRNA targets to find the IncRNA-miRNA interactions. TargetScan (http://www.TargetScan.org/), miRDB (http://www.mirdb. org/) and miRanda (http://www.microrna.org/microrna/home. do) were used to predict miRNA-targeted mRNA. Then, the intersection with the differentially expressed $\operatorname{lnRNAs}$ and mRNAs was retained. Finally, the 1 ncRNA/miRNA/mRNA ceRNA network was constructed using Cytoscape v3.0 (http://www.cytoscape.org/). Fig. 2. reveals the flowchart of the ceRNA network.

GO and pathway analysis. Differentially expressed mRNAs included in the ceRNA network were entered into the Database for Annotation, Visualization, and Integrated Discovery (DAVID) bioinformatics resource (https://david.ncifcrf.gov/) for functional enrichment analysis.

Clinical feature analysis of key lncRNAs. Based on the bioinformatics analysis and the ceRNA network, the relationship between the clinical features, including sex, age, tumour staging, TNM staging and lymphatic metastasis, and the expression of key IncRNAs was analysed. In addition, the association between side-specific lncRNAs and colon cancer patient survival time was analysed.

RNA extraction and reverse transcription-quantitative polymerase chain reaction ( $R T-q P C R)$ validation. RNA from tissue samples was extracted by TRIzol reagent (Invitrogen; Thermo Fisher Scientific, Inc., Waltham, MA, USA). A PrimeScript RT kit (Takara Biotechnology Co., Ltd., Dalian, China) was used to synthesize complementary DNA (cDNA). qPCR was performed in a $20-\mu 1$ volume system ( $2 \mu \mathrm{l}$ cDNA; $1.2 \mu \mathrm{l}$ primers; 


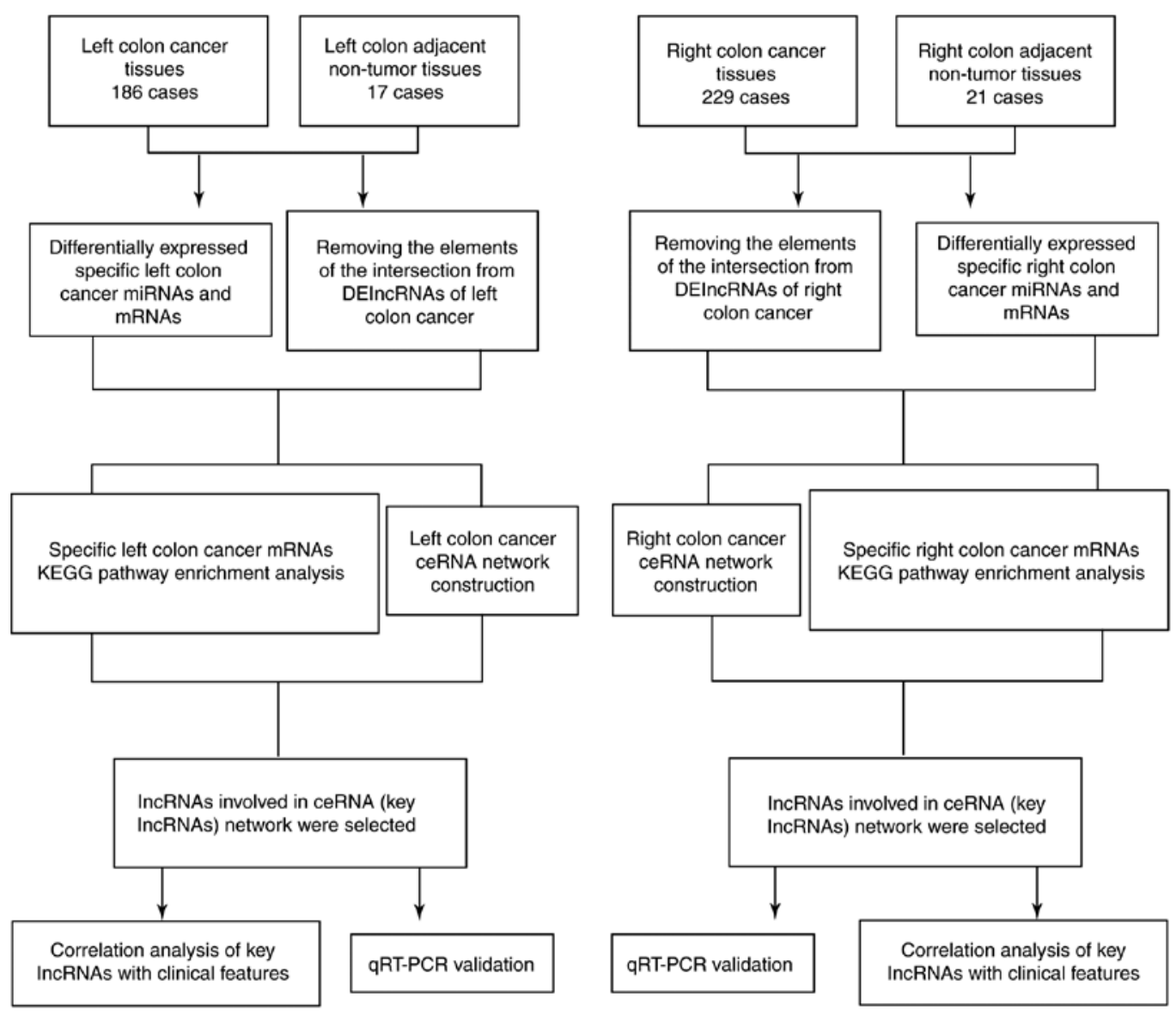

Figure 1. Flowchart of bioinformatics analysis. lncRNAs, long non-coding RNAs; DElncRNAs, differentially expressed lncRNAs; miRNAs, microRNAs; KEGG, Kyoto Encyclopaedia of Genes and Genomes; ceRNAs, competing endogenous RNAs.

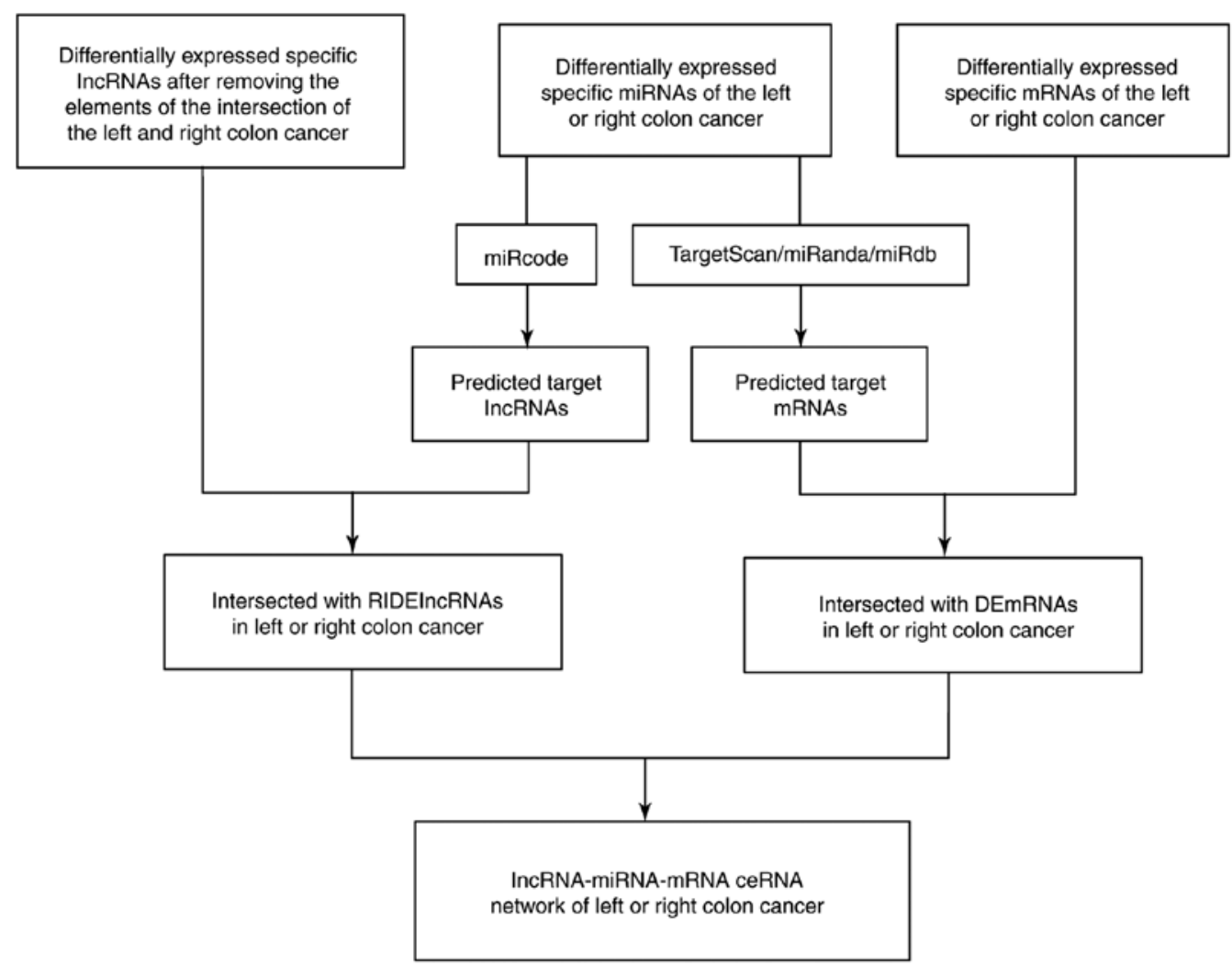

Figure 2. Flowchart of the construction of a ceRNA network. IncRNAs, long non-coding RNAs; DElncRNAs, differentially expressed lncRNAs; RIDElncRNAs, DElncRNAs after removing the elements of the intersection of left and right colon cancer; miRNAs, microRNAs; ceRNAs, competing endogenous RNA 
Table I. Key lncRNAs involved in the ceRNA network.

\begin{tabular}{lcclcr}
\hline $\begin{array}{l}\text { lncRNAs from } \\
\text { left colon cancer }\end{array}$ & $\begin{array}{c}\operatorname{Lg}_{2} \\
\text { (fold change) }\end{array}$ & $\begin{array}{c}\text {-Log } \\
\text { (FDR) }\end{array}$ & $\begin{array}{c}\text { lncRNAs from } \\
\text { right colon cancer }\end{array}$ & $\begin{array}{c}\log _{2} \\
\text { (fold change) }\end{array}$ & $\begin{array}{r}\text {-Log } \\
\text { (FDR) }\end{array}$ \\
\hline CMAHP & -2.31 & 20.48 & LINC00483 & -2.15 & 17.50 \\
PRSS30P & 2.05 & 4.64 & LINC00488 & -3.20 & 15.36 \\
GRIK1-AS1 & -2.66 & 16.87 & LPP-AS1 & 3.94 & 2.06 \\
MIR7-3HG & -3.05 & 15.69 & COL4A2-AS2 & 2.53 & 2.92 \\
WT1-AS & 3.21 & 2.76 & ST7-AS2 & 3.47 & 2.38 \\
MIR22HG & -2.17 & 38.57 & MIR205HG & 5.31 & 2.26 \\
MUC19 & 3.33 & 2.53 & WASIR2 & 3.06 & 6.24 \\
LY86-AS1 & -2.12 & 5.68 & OSBPL10-AS1 & 2.75 & 2.59 \\
LINC00473 & -2.79 & 16.34 & ERVH48-1 & 3.12 & 2.77 \\
LINC00393 & 3.54 & 2.23 & DSCAM-AS1 & 4.55 & 2.11 \\
STEAP2-AS1 & 2.88 & 4.84 & EGOT & 2.28 & 4.27 \\
ATP11A-AS1 & 3.72 & 2.40 & THOC7-AS1 & 2.27 & 2.14 \\
CYP1B1-AS1 & -2.28 & 25.21 & ZBTB20-AS1 & 2.37 & 2.07 \\
BOK-AS1 & 3.63 & AC007731.1 & 3.47 & 3.56 \\
LINC00402 & 5.57 & 19.67 & ITGB5-AS1 & 2.50 & 2.40 \\
AC112721.1 & -3.18 & 3.18 & ARHGEF38-IT1 & 2.21 & 4.51 \\
GDNF-AS1 & 2.84 & 17.19 & ANO1-AS2 & 2.02 & 2.28 \\
ITPK1-AS1 & -2.59 & 2.01 & C8orf49 & 3.46 & 2.29 \\
& 2.38 & & RMST & -2.01 & 3.57 \\
& & & NOVA1-AS1 & -2.01 & 3.48 \\
& & & KCNQ1OT1 & 2.33 & 6.52 \\
\hline
\end{tabular}

lncRNAs, long non-coding RNAs; ceRNA, competing endogenous RNA.

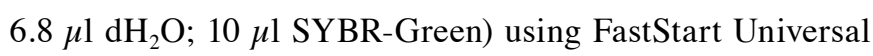
SYBR-Green Master Kit (Roche Diagnostics, Indianapolis, IN, USA) and a StepOnePlus Real-time PCR System (Applied Biosystems; Thermo Fisher Scientific, Inc.). Thermocycling conditions were as follows: Hot-start DNA polymerase activation at $95^{\circ} \mathrm{C}$ for $10 \mathrm{~min}, 40$ cycles at $95^{\circ} \mathrm{C}$ for $15 \mathrm{sec}$ and $60^{\circ} \mathrm{C}$ for $1 \mathrm{~min}$, followed by one cycle of melt curve analysis at $95^{\circ} \mathrm{C}$ for $15 \mathrm{sec}, 60^{\circ} \mathrm{C}$ for $1 \mathrm{~min}$, and $95^{\circ} \mathrm{C}$ for $15 \mathrm{sec}$. The data were analysed using the $2^{-\Delta \Delta \mathrm{Cq}}(14)$ method and the mRNA expression levels were normalized to GAPDH. The rimer sequences were as follows: LINC00402 forward, 5'-TAGGCAGGAAAG AGGTTG-3' and reverse, 5'-TGGTAGGTAGCAGGTGGT-3'; KCNQ1OT1 forward, 5'-AGGGTGACAGTGTTTCATAGG CT-3' and reverse, 5'-GAGGCACATTCATTCGTTGGT-3'; GAPDH forward, 5'-ACAGTCAGCCGCATCTTCTT-3' and reverse, 5'-GACAAGCTTCCCGTTCTCAG-3'. All RT-qPCR reactions were performed in triplicate.

Statistical analysis. All the results were expressed as the mean \pm SD. R Studio (R version 3.4.1; https://www.rstudio. com), Statistical Programme for Social Sciences 20.0 (IBM Corp., Armonk, NY, USA) and GraphPad Prism 5.0 software (GraphPad Software, Inc., La Jolla, CA, USA) were used to analyse the data. The prognostic characteristics of IncRNAs based on the univariate Cox proportional hazards regression model were detected. Then, multivariate Cox regression analysis was applied for further study. The differences in the
qRT-PCR results were compared by paired Student's t-test. $\mathrm{P}<0.05$ was considered to indicate a statistically significant difference.

\section{Results}

DElncRNAs that are included exclusively in left or right colon cancer. It was determined that 879 lncRNAs (DElncRNA) were differentially expressed between tumour samples and adjacent tissues in left colon cancer from TCGA database [absolute $\log _{2}$ (fold change) $\left.>2.0, \mathrm{FDR}<0.01\right]$. There were 916 in right colon cancer. After removing the elements of the intersection from DElncRNAs (RIDElncRNAs) of left and right colon cancer, the DElncRNAs included exclusively in left or right colon cancer were obtained, including 277 DElncRNAs (RIDElncRNAs) in the left colon cancer and 314 DElncRNAs (RIDElncRNAs) in the right colon cancer. Finally, 18 RIDElncRNAs were used to construct the ceRNA network of left colon cancer (Tables I and II), and 21 RIDElncRNAs were used to construct the network of right colon cancer (Tables I and III).

Prediction of IncRNA-targeted miRNAs. For further investigation, 165 and 227 side-specific miRNAs that were differentially expressed between tumour tissues and adjacent tissues from left and right colon cancer, respectively, were determined. These miRNAs were identified as side-specific 
Table II. lncRNAs that may target specific miRNAs in left colon cancer.

\begin{tabular}{|c|c|}
\hline lncRNAs & miRNAs \\
\hline СМАНР & $\begin{array}{l}\text { hsa-mir-96, hsa-mir-141, hsa-mir-144, hsa-mir-145, hsa-mir-150, hsa-mir-424, hsa-mir-182, hsa-mir-183, } \\
\text { hsa-mir-187, hsa-mir-192, hsa-mir-215, hsa-mir-375 }\end{array}$ \\
\hline PRSS30P & hsa-mir-143, hsa-mir-150, hsa-mir-424, hsa-mir-21 \\
\hline GRIK1-AS1 & hsa-mir-145, hsa-mir-375 \\
\hline MIR7-3HG & hsa-mir-145, hsa-mir-150 \\
\hline WT1-AS & $\begin{array}{l}\text { hsa-mir-96, hsa-mir-141, hsa-mir-145, hsa-mir-424, hsa-mir-17, hsa-mir-182, hsa-mir-98, hsa-mir-193b, } \\
\text { hsa-mir-429, hsa-mir-22, hsa-mir-32, hsa-mir-375 }\end{array}$ \\
\hline MIR22HG & hsa-mir-375, hsa-mir-32 \\
\hline MUC19 & $\begin{array}{l}\text { hsa-mir-375, hsa-mir-22, hsa-mir-32, hsa-mir-429, hsa-mir-193b, hsa-mir-215, hsa-mir-192, hsa-mir-187, } \\
\text { hsa-mir-98, hsa-mir-182, hsa-mir-17, hsa-mir-424, hsa-mir-150, hsa-mir-152, hsa-mir-14, hsa-mir-144, } \\
\text { hsa-mir-143, hsa-mir-96, hsa-mir-454 }\end{array}$ \\
\hline LY86-AS1 & $\begin{array}{l}\text { hsa-mir-375, hsa-mir-187, hsa-mir-183, hsa-mir-182, hsa-mir-424, hsa-mir-150, hsa-mir-145, hsa-mir-141, } \\
\text { hsa-mir-96, hsa-mir-454 }\end{array}$ \\
\hline LINC00473 & hsa-mir-424, hsa-mir-150, hsa-mir-145 \\
\hline LINC00393 & hsa-mir-215, hsa-mir-192 \\
\hline STEAP2-AS1 & hsa-mir-375, hsa-mir-424, hsa-mir-152, hsa-mir-143 \\
\hline ATP11A-AS1 & hsa-mir-22, hsa-mir-215, hsa-mir-192, hsa-mir-187, hsa-mir-424, hsa-mir-152, hsa-mir-143, hsa-mir-96 \\
\hline CYP1B1-AS1 & hsa-mir-21, hsa-mir-429, hsa-mir-193b, hsa-mir-150, hsa-mir-152, hsa-mir-145, hsa-mir-454 \\
\hline BOK-AS1 & hsa-mir- 150 \\
\hline LINC00402 & hsa-mir-22, hsa-mir-429, hsa-mir-193b, hsa-mir-182, hsa-mir-17, hsa-mir-150, hsa-mir-143, hsa-mir-141 \\
\hline AC112721.1 & hsa-mir-424 \\
\hline GDNF-AS1 & hsa-mir-187, hsa-mir-424, hsa-mir-145, hsa-mir-143 \\
\hline ITPK1-AS1 & hsa-mir-22, hsa-mir-17, hsa-mir-150, hsa-mir-144, hsa-mir-141 \\
\hline
\end{tabular}

lncRNAs, long non-coding RNAs; miRNAs, microRNAs.

miRNAs. Then, it was determined whether these 165 miRNAs could target the aforementioned 277 RIDElncRNAs in left colon cancer and whether the 227 miRNAs could target the 314 RIDElncRNAs in right colon cancer. Based on miRcode (http://www.mircode.org/), 22 miRNAs targeting 18 lncRNAs were predicted in left colon cancer (Table II), and there were 27 miRNAs and 21 lncRNAs predicted in the ceRNA of right colon cancer (Table III).

Prediction of miRNA-targeted mRNAs. First, 2,028 and 2,069 differentially expressed mRNAs between tumour tissues and adjacent tissues [absolute $\log _{2}$ (fold change) $>2$, FDR $<0.01]$ in left and right colon cancer, respectively, were identified. These mRNAs were identified as side-specific mRNAs (DEmRNAs). The 2,028 and 2,069 DEmRNAs were analysed with DAVID bioinformatics resources. According to the P-values, the top $21 \mathrm{KEGG}$ pathways of the DEmRNAs were revealed (Figs. 3 and 4). Among these pathways, the transcriptional dysregulation in cancer, cAMP, Wnt and PPAR signalling pathways, which were related to cancer-associated pathways, were revealed to be more important in left colon cancer. However, the chemical carcinogenesis pathway was more involved in right colon cancer. For further investigation, the miRNAs included in the ceRNA network were then used to predict the targeted mRNAs using miRanda, TargetScan and miRDB. The intersection of the predicted mRNAs and
DEmRNAs was obtained. Finally, 57 mRNAs were included in the left colon cancer ceRNA network, and 55 were included in the right (Tables IV and V).

According to the relationship between RNAs revealed by Tables II-V, IncRNA-miRNA-mRNA ceRNA networks were constructed. Cytoscape 3.0 was used to draw the ceRNA network. In our results, there were 18 lncRNAs, 22 miRNAs and 57 mRNAs included in the left colon cancer ceRNA network and 21 lncRNAs, 27 miRNAs and 55 mRNAs included in the right ceRNA network (Figs. 5 and 6).

Key IncRNAs and clinical feature association. The clinical features of the key lncRNAs in the ceRNA network were then further analysed. The clinical features, including sex, tumour grade, TNM stage and lymphatic metastasis status, were provided by TCGA data sets. In total 15 lncRNAs were revealed, including 3 in left colon cancer and 12 in right colon cancer, which were significantly related to clinical features (Table VI). The results revealed that CYP1B1-AS1, LPP-AS1, MIR205HG, DSCAM-AS1, RMST and NOVA1-AS1 were related to age, LPP-AS1, WASIR2, OSBPL10-AS1 and DSCAM-AS1 were related to sex, AC112721.1, DSCAM-AS1, ARHGEF38-IT1, C8orf49 and KCNQ1OT1 were related to lymphatic metastasis, LINC00402 and ZBTB20-AS1 were related to tumour grade, and AC112721.1, DSCAM-AS1, ARHGEF38-IT1, C8orf49 and KCNQ1OT1 were related to TNM stage (Table VI). 
Table III. IncRNAs that may target specific miRNAs in right colon cancer.

IncRNAs miRNAs

LINC00483 hsa-mir-223, hsa-mir-21, hsa-mir-215, hsa-mir-192, hsa-mir-183, hsa-mir-182, hsa-mir-17, hsa-mir-150, hsa-mir-144, hsa-mir-96, hsa-mir-106a

LINC00488 hsa-mir-21, hsa-mir-215, hsa-mir-192, hsa-mir-98, hsa-mir-144, hsa-mir-96

LPP-AS1 hsa-mir-338, hsa-mir-143

COL4A2-AS2 hsa-mir-338, hsa-mir-424, hsa-mir-150, hsa-mir-152

ST7-AS2

MIR205HG hsa-mir-22, hsa-mir-429, hsa-mir-215, hsa-mir-192, hsa-mir-145

hsa-mir-22, hsa-mir-215, hsa-mir-192, hsa-mir-183, hsa-mir-150, hsa-mir-152, hsa-mir-145, hsa-mir-143, hsa-mir-454, hsa-mir-301b

WASIR2 hsa-mir-338, hsa-mir-193b, hsa-mir-150

OSBPL10-AS1 hsa-mir-375, hsa-mir-182, hsa-mir-424, hsa-mir-145, hsa-mir-96

ERVH48-1 hsa-mir-338, hsa-mir-223, hsa-mir-22, hsa-mir-21, hsa-mir-187, hsa-mir-98, hsa-mir-182, hsa-mir-145, hsa-mir-144, hsa-mir-141, hsa-mir-96, hsa-mir-454, hsa-mir-301b

DSCAM-AS1 hsa-mir-338, hsa-mir-150, hsa-mir-143, hsa-mir-141

EGOT

THOC7-AS 1

ZBTB20-AS1

AC007731.1

ITGB5-AS1

ARHGEF38-IT1

ANO1-AS2

C8orf49

hsa-mir-375, hsa-mir-21, hsa-mir-183, hsa-mir-424, hsa-mir-143, hsa-mir-141

hsa-mir-215, hsa-mir-192, hsa-mir-187

hsa-mir-217, hsa-mir-152, hsa-mir-454, hsa-mir-301b

hsa-mir-215, hsa-mir-192, hsa-mir-183, hsa-mir-150, hsa-mir-152

hsa-mir-21, hsa-mir-193b

hsa-mir-338, hsa-mir-150, hsa-mir-143

hsa-mir-375, hsa-mir-98, hsa-mir-17, hsa-mir-152, hsa-mir-106a

hsa-mir-375, hsa-mir-338, hsa-mir-32, hsa-mir-429, hsa-mir-17, hsa-mir-424, hsa-mir-150, hsa-mir-152, hsa-mir-143, hsa-mir-106a, hsa-mir-454, hsa-mir-301b

RMST hsa-mir-375, hsa-mir-338, hsa-mir-32, hsa-mir-429, hsa-mir-193b, hsa-mir-182, hsa-mir-17, hsa-mir-424, hsa-mir-150, hsa-mir-145, hsa-mir-144, hsa-mir-96, hsa-mir-454, hsa-mir-301b

NOVA1-AS1 hsa-mir-223, hsa-mir-22, hsa-mir-217

KCNQ1OT1 hsa-mir-375, hsa-mir-338, hsa-mir-32, hsa-mir-223, hsa-mir-22, hsa-mir-217, hsa-mir-429, hsa-mir-193b, hsa-mir-215, hsa-mir-192, hsa-mir-187, hsa-mir-98, hsa-mir-183, hsa-mir-301b, hsa-mir-454, hsa-mir-106a, hsa-mir-96, hsa-mir-141, hsa-mir-143, hsa-mir-145, hsa-mir-152, hsa-mir-150, hsa-mir-182, hsa-mir-17, hsa-mir-424

lncRNAs, long non-coding RNAs; miRNAs, microRNAs.

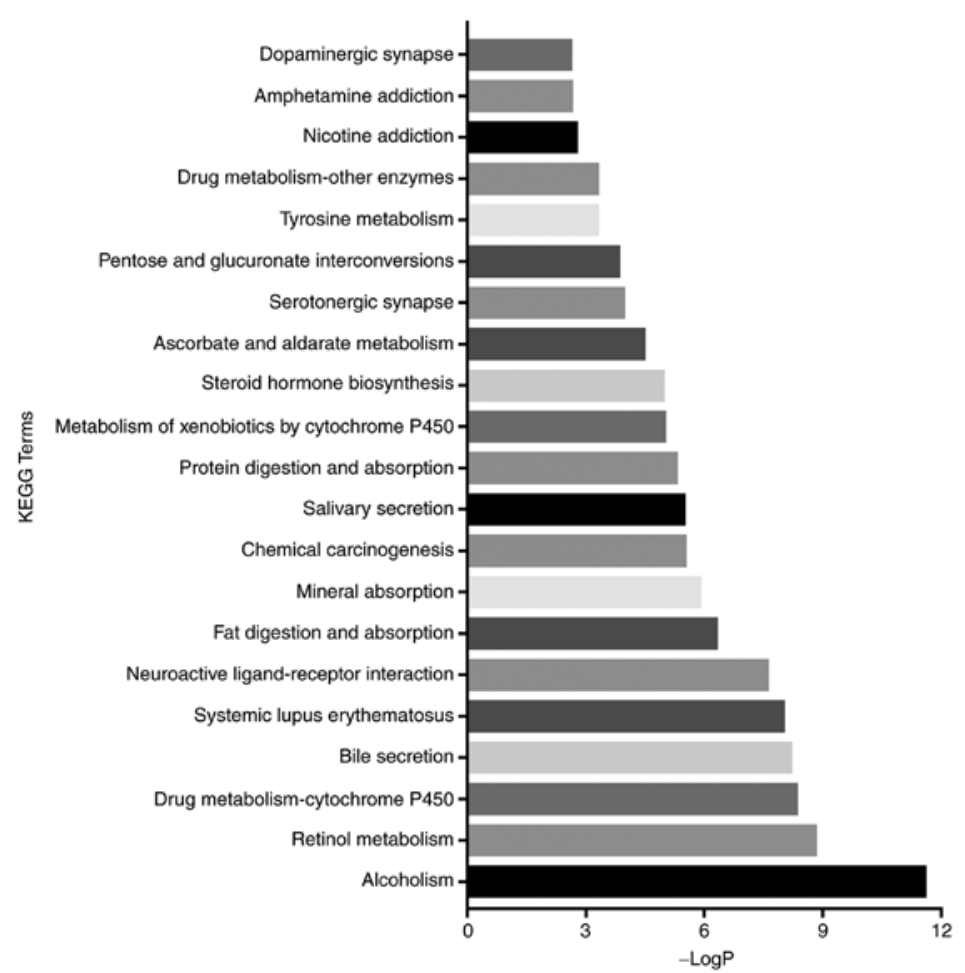

Figure 3. Top 21 KEGG terms for mRNAs from the left colon cancer. -LogP, -Log(P-value); KEGG, Kyoto Encyclopaedia of Genes and Genomes. 
Table IV. miRNAs that may target specific mRNAs in left colon cancer.

\begin{tabular}{ll}
\hline miRNAs & \\
\hline hsa-mir-141 & PHLPP2, ELAVL4, MACC1, KIAA1549, EPHA7 \\
hsa-mir-143 & COL1 1, SERPINE1 \\
hsa-mir-144 & GRIK3, TBX18 \\
hsa-mir-145 & SERPINE1 \\
hsa-mir-150 & HILPDA, SLC7A11, ZNF460, EREG \\
hsa-mir-152 & NPTX1, BMP3, KLF4 \\
hsa-mir-17 & E2F1, FOXQ1, CADM2, FJX1, CFL2, SALL3, FAM129A, SMOC1, CLIP4, FAM46C, ANKRD33B \\
hsa-mir-182 & CHL1, NPTX1, TCEAL7, FOXF2, ULBP2 \\
hsa-mir-183 & KIF5C \\
hsa-mir-192 & TCF7 \\
hsa-mir-193b & PLAU, DCAF7 \\
hsa-mir-21 & EDIL3, OSR1, ATP2B4, TGFBI \\
hsa-mir-215 & TCF7 \\
hsa-mir-22 & RGS2 \\
hsa-mir-32 & UGP2, CCDC113, PHLPP2, ATP2B4 \\
hsa-mir-375 & ELAVL4 \\
hsa-mir-424 & PSAT1, PHLPP2, ZNRF3, TPM2, TMEM100, AXIN2, CBX2 \\
hsa-mir-454 & RBM20, SALL3, CFL2, SMOC1 \\
hsa-mir-96 & ALK, TRIB3 \\
hsa-mir-98 & TRIM71, CPA4, IGF2BP3, HAND1, SLC5A6, PRSS22, IGF2BP1 \\
\hline
\end{tabular}

miRNAs, microRNAs.

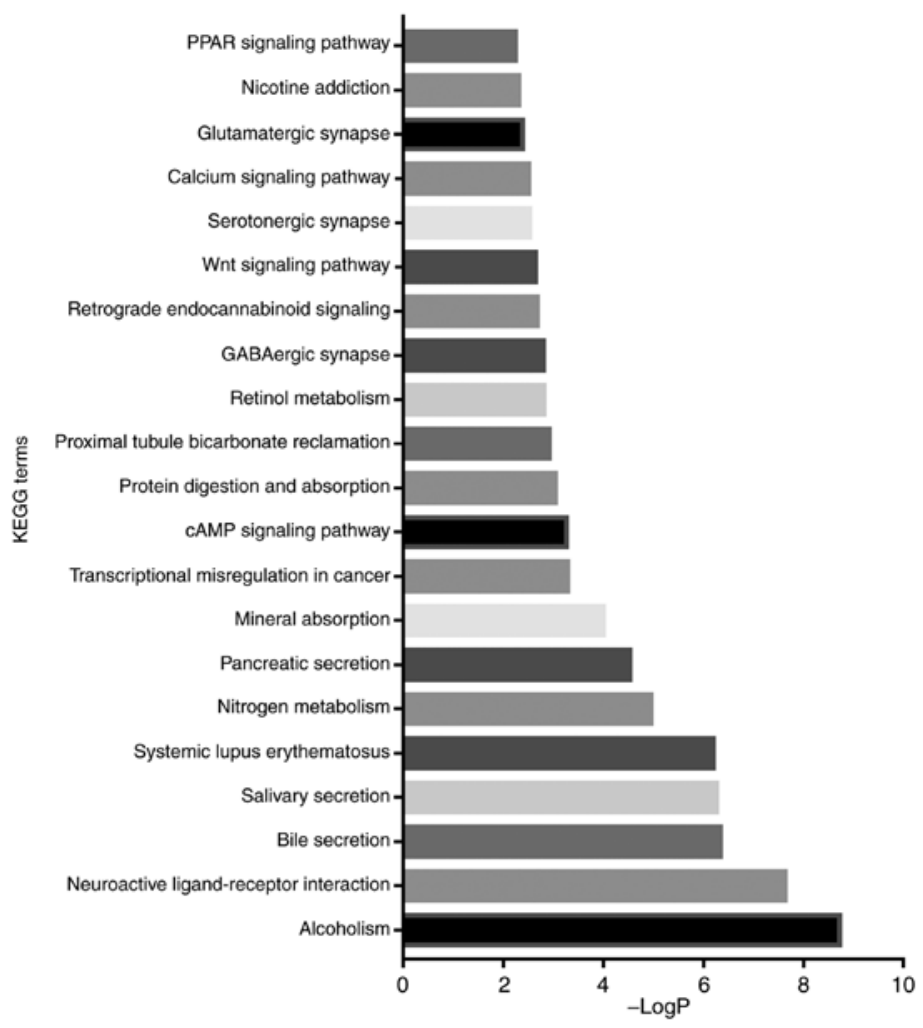

Figure 4. Top 21 KEGG terms for mRNAs from the right colon cancer. -LogP, - $\log (\mathrm{P}-\mathrm{value})$; KEGG, Kyoto Encyclopaedia of Genes and Genomes.

Furthermore, the prognostic characteristics of RIDElncRNAs from both sides were detected. Based on the univariate Cox proportional hazards regression model, the association of the overall survival of patients with the expression 
Table V. miRNAs that may target specific mRNAs in right colon cancer.

miRNAs mRNAs

hsa-mir-106a

hsa-mir-141

hsa-mir-143

hsa-mir-144

hsa-mir-145

hsa-mir- 150

hsa-mir-152

hsa-mir-17

hsa-mir-182

hsa-mir-183

hsa-mir-192

hsa-mir-193b

hsa-mir-21

hsa-mir-217

hsa-mir-223

hsa-mir-32

hsa-mir-338

hsa-mir-375

hsa-mir-424

hsa-mir-429

hsa-mir-454

hsa-mir-96

hsa-mir-98
CFL2, FAM129A, FJX1, CADM2, FOXQ1

KIAA1549, PHLPP2, ELAVL4, EPHA7, DPY19L1

COL1A1, SERPINE1

GRIK3, KCNQ5

SERPINE1

ZNF460, PDCD4, SLC7A11, HILPDA, EREG

KLF4, NPTX1, BMP3

FOXQ1, CADM2, FJX1, FAM129A, CFL2, SLC16A9, CYBRD1

ULBP2, CHL1, NPTX1, FOXF2

PDCD4

GRHL1

PLAU

PDCD4, TGFBI, JPH1

DACH1

ECT2, EPB41L3

PAX9, PBLD, MIER3, PHLPP2, UGP2

NOVA1

ELAVL4

TPM2, TMEM100, PHLPP2, CBX2, E2F7, PSAT1, AXIN2

PMAIP1

CFL2, RBM20

TRIB3

RGS16, IGF2BP3, TRIM71, PRSS22, HAND1, IGF2BP1, FMO4, CPA4

miRNAs, microRNAs.

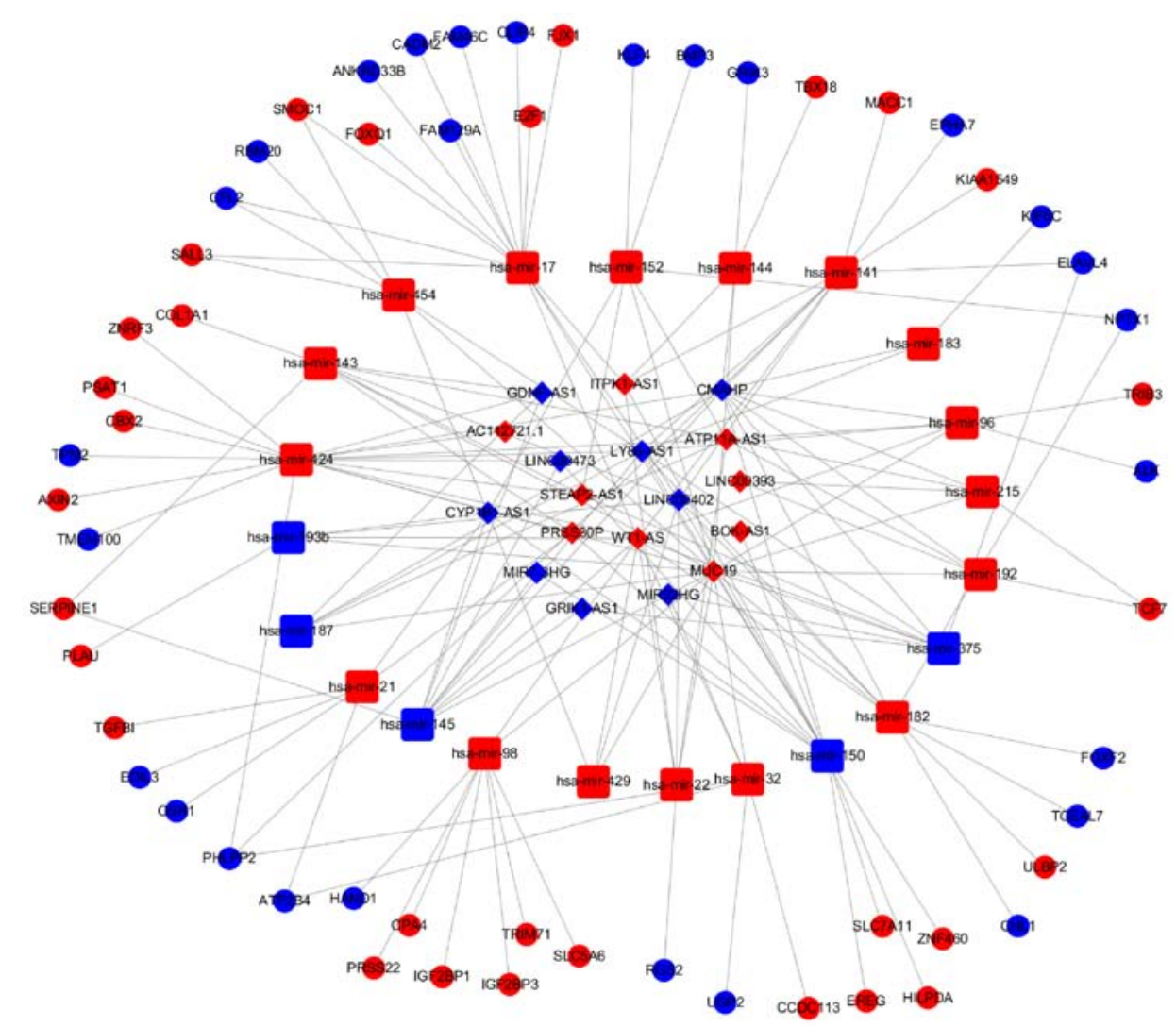

Figure 5. The lncRNA/miRNA/mRNA ceRNA network of left colon cancer. Red balls, upregulated mRNAs; blue balls, downregulated mRNAs; red squares, upregulated miRNAs; blue squares, downregulated miRNAs; red diamonds, upregulated lncRNAs; blue diamonds, downregulated lncRNAs. IncRNAs, long non-coding RNAs; miRNAs, microRNAs; ceRNA, competing endogenous RNA. 
Table VI. Correlation between key lncRNAs involved in the ceRNA network and their clinical features.

\begin{tabular}{|c|c|c|}
\hline Comparisons & Left colon cancer & Right colon cancer \\
\hline Age (<60 vs. $>60$ years $)$ & CYP1B1-AS1 & $\begin{array}{l}\text { LPP-AS1, MIR205HG, DSCAM-AS1, RMST, } \\
\text { NOVA1-AS1 }\end{array}$ \\
\hline Sex (female vs. male) & & LPP-AS1, WASIR2, OSBPL10-AS1, DSCAM-AS1 \\
\hline Lymphatic metastasis (no vs. yes) & AC112721.1 & DSCAM-AS1,ARHGEF38-IT1, C8orf49, KCNQ1OT1 \\
\hline TNM staging system (T1+T2 vs. T3+T4) & LINC00402 & ZBTB20-AS1 \\
\hline Tumour stage (stage I, II vs. stage III, IV) & AC112721.1 & DSCAM-AS1, ARHGEF38-IT1, C8orf49, KCNQ1OT1 \\
\hline MSI status (MSI-H vs. other status) & & $\begin{array}{l}\text { LINC00483, LINC00488, WASIR2, DSCAM-AS1, } \\
\text { NOVA1-AS1 }\end{array}$ \\
\hline
\end{tabular}

lncRNAs, long non-coding RNAs; ceRNA, competing endogenous RNA.

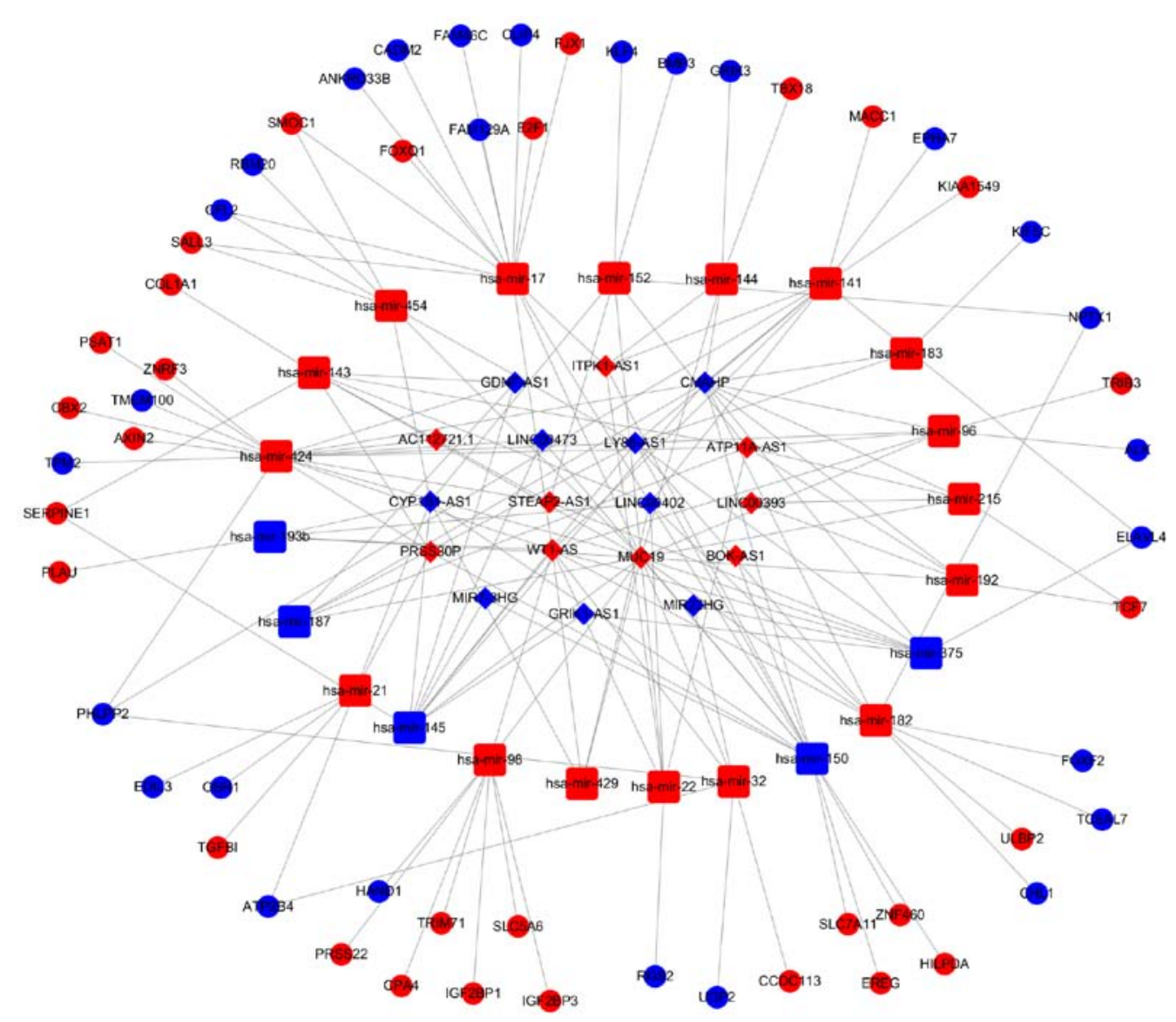

Figure 6. The lncRNA/miRNA/mRNA ceRNA network of right colon cancer. Red balls, upregulated mRNAs; blue balls, downregulated mRNAs; red squares, upregulated miRNAs; blue squares, downregulated miRNAs; red diamonds, upregulated lncRNAs; blue diamonds, downregulated lncRNAs. IncRNAs, long non-coding RNAs; miRNAs, microRNAs; ceRNA, competing endogenous RNA.

level of RIDElncRNAs was analysed. Finally, 20 lncRNAs from left colon cancer that were significantly associated with overall survival (log-rank $\mathrm{P}<0.05)$ (Fig. S1 and Table VII) were revealed, and 25 lncRNAs from right colon cancer were determined to be related to overall survival (log-rank $\mathrm{P}<0.05$ ) (Fig. S2 and Table VII). Multivariate Cox regression analysis was then performed with these lncRNAs. Fifteen lncRNAs from the left colon cancer were revealed to be independent factors of survival time and 12 in the right colon cancer. The results are presented in Table VIII.
Subsequently, to confirm the reliability of the bioinformatics results, 2 key lncRNAs (LINC00402 from the left side and KCNQ1OT1 from the right side) were randomly selected from the networks and their RNA expression level was determined in 58 paired colon cancer tissues from left colon cancer and right colon cancer. The bioinformatics results revealed that LINC00402 presented lower expression in left colon cancer tissues than adjacent non-tumour tissues, and KCNQ1OT1 was significantly expressed higher in tumour tissues. Fig. 7 revealed that the qRT-PCR results were consistent with the 
Table VII. Kaplan-Meier survival analysis for lncRNAs that were associated with overall survival.

\begin{tabular}{llll}
\hline $\begin{array}{l}\text { lncRNAs } \\
\text { from the left }\end{array}$ & P-value & $\begin{array}{c}\text { lncRNAs } \\
\text { from the right }\end{array}$ & P-value \\
\hline AC019118.4 & 0.003 & AC003991.3 & 0.005 \\
CTB-181H17.1 & 0.01 & AC011288.2 & 0.003 \\
CTD-2308L22.1 & 0.04 & AC012531.25 & 0.01 \\
FGF13-AS1 & 0.006 & B4GALT1-AS1 & 0.0005 \\
HORMAD2-AS1 & 0.03 & CTC-428G20.6 & 0.02 \\
IGBP1-AS2 & 0.03 & CTC-573N18.1 & 0.05 \\
LINC01990 & 0.02 & CTD-2591A5.2 & 0.05 \\
RP1-10C16.1 & 0.03 & CTD-3064M3.7 & 0.05 \\
RP11-108K3.1 & 0.04 & LINC00513 & 0.005 \\
RP11-205M3.3 & 0.03 & LINC01630 & 0.03 \\
RP11-281P23.2 & 0.002 & LINC01633 & 0.008 \\
RP11-342A23.2 & 0.01 & RP11-126H7.4 & 0.04 \\
RP11-354P11.4 & 0.02 & RP11-138A9.1 & 0.04 \\
RP11-475B2.1 & 0.03 & RP11-138A9.2 & 0.01 \\
RP11-515O17.2 & 0.01 & RP11-157F20.3 & 0.03 \\
RP11-674E16.4 & 0.02 & RP11-277P12.20 & 0.05 \\
RP11-686O6.1 & 0.04 & RP11-304L19.12 & 0.03 \\
RP11-713P17.3 & 0.01 & RP11-495P10.5 & 0.002 \\
RP3-453C12.15 & 0.04 & RP11-619I22.1 & 0.03 \\
RP6-114E22.1 & 0.02 & RP11-661A12.9 & 0.04 \\
& & RP11-67K19.3 & 0.05 \\
& & RP1-29C18.10 & 0.05 \\
& & RP4-676L2.1 & 0.05 \\
& & RP4-811H24.9 & 0.05 \\
& & & 0.03 \\
\hline
\end{tabular}

lncRNAs, long non-coding RNAs.

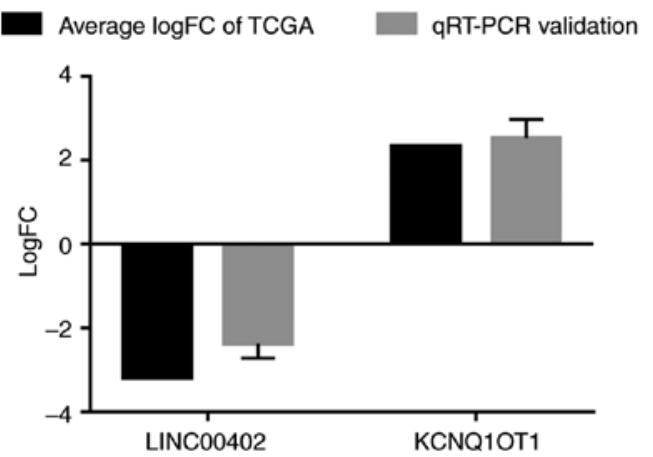

Figure 7. Comparison of $\log \mathrm{FC}(-\Delta \Delta \mathrm{Cq})$ of $\operatorname{lncRNAs}$ between TCGA and qRT-PCR results. $\mathrm{Log} F C, \log _{2}$ (fold change).

bioinformatics results. The relationship between the expression of the 2 lncRNAs and clinicopathological characteristics was then further analysed. The results revealed that LINC00402 was related to TNM staging (Table IX), and KCNQ1OT1 was significantly associated with lymphatic metastasis and tumour stage (Table IX), which were almost the same as the bioinformatics results (Table VI).

\section{Discussion}

In terms of clinical behaviour and response to therapy, colorectal cancer $(\mathrm{CRC})$ is a heterogeneous disease $(15,16)$. Patients can benefit from this heterogeneity when stratified for their response to therapeutic strategies. However, CRC development involves the complex transformation of molecular events of which we lack enough knowledge. CRC still has a high incidence and mortality. It is our hope that the study of the molecular difference between left and right side colon cancer will help with the exploration of CRC heterogeneity.

In the present study, we determined side-specific IncRNAs, miRNAs and mRNAs based on the differential expression between tumour tissues and adjacent non-tumour tissues in the two sides. Through KEGG analysis, we analysed the pathways that the side-specific mRNAs may be involved in. Combining the bioinformatics resources, we established ceRNA networks with side-specific DEmRNAs, DEmiRNAs and RIDElncRNAs. We then further analysed the clinical features of the key lncRNAs belonging to the ceRNA network. Side-specific RIDElncRNAs were further analysed to determine whether they were correlated with overall survival. To check the reliability of the bioinformatics results, we randomly selected 2 key lncRNAs (LINC00402 from the left and KCNQ1OT1 from the right) and determined their expression by qRT-PCR.

There were several cancer-related roles in the RIDElncRNA group from the two sides. For example, MIR22HG was reported to suppressed hepatocellular and endometrial carcinoma $(17,18)$ and linc 00483 was reported to promoted gastric cancer (19). We conducted univariate and multivariate Cox regression analyses and found that $15 \mathrm{lncRNAs}$ from the left and 12 lncRNAs from the right were found to be independent factors of survival time (Table VIII). In KEGG analysis, we identified the top 21 pathways of the DEmRNAs (Figs. 3 and 4). After removing the same KEGG terms, the results revealed that different cancer-related pathways were involved in the two sides, i.e., the transcriptional dysregulation in cancer, cAMP, Wnt and PPAR signalling pathways were more important in the left colon cancer, and the chemical carcinogenesis pathway played a more important role in the right colon cancer. Some of these cancer-related pathways, such as cAMP, Wnt and PPAR signalling pathways, have been reported to play important roles in the CRC. Lu et al reported that the cAMP pathway could be activated to inhibit angiogenesis and vasculogenic mimicry in CRC (20). As previous studies reported $(21,22)$, the Wnt pathway could reduce apoptosis, stimulate cell proliferation and promote metastasis in CRC. Zarkou et al revealed that IncRNAs can modulate the WNT pathway by affecting gene expression through diversified mechanisms, from the transcriptional to the post-translational level (23). As for the PPAR signalling pathway, it has been revealed to be inhibited in CRC (24). However, few studies have examined the performance of these pathways in left or right colon cancer.

By constructing the ceRNA network, our research focused on the potentially different mechanisms of distal and proximal colon cancers. Several previous studies have already reported the interactions between RNAs in the ceRNA network. For example, MIR22HG, an lncRNA from the left ceRNA network, was reported to interact with miR-141-3p 
Table VIII. Results of multivariate cox regression analysis.

\begin{tabular}{|c|c|c|c|c|c|c|c|}
\hline $\begin{array}{l}\text { lncRNAs } \\
\text { from the left }\end{array}$ & $\beta$ & $\begin{array}{c}\text { OR } \\
(95 \% \mathrm{CI})\end{array}$ & P-value & $\begin{array}{l}\text { lncRNAs } \\
\text { from the right }\end{array}$ & $\beta$ & $\begin{array}{c}\text { OR } \\
(95 \% \mathrm{CI})\end{array}$ & P-value \\
\hline RP6-114E22.1 & -1.037 & $0.354(0.158-0.793)$ & 0.012 & AC003991.3 & 0.676 & $1.966(1.142-3.387)$ & 0.015 \\
\hline RP3-453C12.15 & -0.515 & $0.597(0.273-1.306)$ & 0.197 & AC011288.2 & -0.706 & $0.494(0.286-0.851)$ & 0.011 \\
\hline RP11-713P17.3 & -0.886 & $0.412(0.188-0.904)$ & 0.027 & AC012531.25 & -0.672 & $0.511(0.287-0.908)$ & 0.022 \\
\hline RP11-686O6.1 & 0.809 & $2.245(1.055-4.778)$ & 0.036 & B4GALT1-AS1 & 0.879 & $2.408(1.347-4.305)$ & 0.003 \\
\hline RP11-674E16.4 & 0.684 & $1.982(0.899-4.369)$ & 0.09 & CTC-428G20.6 & 0.413 & $1.511(0.869-2.628)$ & 0.144 \\
\hline RP11-515O17.2 & 1.404 & $4.072(1.591-10.424)$ & 0.003 & CTC-573N18.1 & -0.543 & $0.581(0.335-1.007)$ & 0.053 \\
\hline RP11-475B2.1 & -0.931 & $0.394(0.179-0.865)$ & 0.02 & CTD-2591A5.2 & -0.708 & $0.492(0.280-0.866)$ & 0.014 \\
\hline RP11-354P11.4 & 1.049 & $2.854(1.252-6.506)$ & 0.013 & CTD-3064M3.7 & -0.541 & $0.582(0.336-1.009)$ & 0.054 \\
\hline RP11-342A23.2 & -0.916 & $0.400(0.182-0.877)$ & 0.022 & LINC00513 & 0.641 & $1.898(1.079-3.337)$ & 0.026 \\
\hline RP11-281P23.2 & 1.023 & $2.783(1.213-6.384)$ & 0.016 & LINC01630 & 0.462 & $1.587(0.896-2.811)$ & 0.113 \\
\hline RP11-205M3.3 & 0.79 & $2.204(1.016-4.780)$ & 0.045 & LINC01633 & -0.702 & $0.496(0.285-0.863)$ & 0.013 \\
\hline RP11-108K3.1 & -0.545 & $0.580(0.255-1.318)$ & 0.193 & RP11-126H7.4 & -0.372 & $0.689(0.398-1.194)$ & 0.184 \\
\hline RP1-10C16.1 & -0.921 & $0.398(0.180-0.879)$ & 0.023 & RP11-138A9.1 & 0.421 & $1.524(0.887-2.619)$ & 0.127 \\
\hline LINC01990 & -0.761 & $0.467(0.208-1.051)$ & 0.066 & RP11-138A9.2 & 0.728 & $2.070(1.150-3.729)$ & 0.015 \\
\hline IGBP1-AS2 & 0.737 & $2.089(0.904-4.828)$ & 0.085 & RP11-157F20.3 & -0.495 & $0.610(0.349-1.064)$ & 0.081 \\
\hline HORMAD2-AS1 & -1.334 & $0.263(0.114-0.607)$ & 0.002 & RP11-277P12.20 & -0.478 & $0.620(0.358-1.073)$ & 0.088 \\
\hline FGF13-AS1 & -1.154 & $0.315(0.143-0.695)$ & 0.004 & RP11-304L19.12 & -0.47 & $0.625(0.365-1.069)$ & 0.086 \\
\hline CTD-2308L22.1 & -1.085 & $0.338(0.147-0.774)$ & 0.01 & RP11-495P10.5 & -0.693 & $0.500(0.282-0.886)$ & 0.017 \\
\hline СТВ-181H17.1 & -1.03 & $0.357(0.160-0.795)$ & 0.012 & RP11-619I22.1 & 0.578 & $1.783(1.048-3.035)$ & 0.033 \\
\hline \multirow[t]{6}{*}{ AC019118.4 } & -0.991 & $0.371(0.170-0.810)$ & 0.013 & RP11-661A12.9 & -0.32 & $0.726(0.417-1.265)$ & 0.259 \\
\hline & & & & RP11-67K19.3 & 0.655 & $1.926(1.103-3.363)$ & 0.021 \\
\hline & & & & RP1-29C18.10 & -0.455 & $0.634(0.370-1.086)$ & 0.097 \\
\hline & & & & RP4-676L2.1 & 0.505 & $1.656(0.924-2.969)$ & 0.09 \\
\hline & & & & RP4-811H24.9 & -0.385 & $0.681(0.394-1.176)$ & 0.168 \\
\hline & & & & SSTR5-AS1 & 0.615 & $1.850(1.061-3.225)$ & 0.03 \\
\hline
\end{tabular}

lncRNAs, long non-coding RNAs.

and therefore inhibited endometrial carcinoma cell proliferation (18). Additionally, KCNQ1OT1, which belonged to the right ceRNA network, modulated CCNE2 by sponging miR-145 in BRCA (25). Another study reported that KCNQ1OT1 could ameliorate particle-induced osteolysis by inhibiting miR-21a-5p (26). These previous studies strongly demonstrated that our analysis was reliable. Therefore, there may be some internal contact between lncRNA/miRNA/mRNA in the progression and development of CRC. Based on significant differences in lncRNA, miRNA and mRNA expression data, a ceRNA network was constructed by bioinformatics prediction and correlation analysis. The relationship between the key lncRNAs and clinical features was then further analysed. In the left ceRNA network, 3 key lncRNAs (CYP1B1-AS1, LINC00402, and AC112721.1) were confirmed to be related to clinical features. AC112721.1 has been reported to be correlated with bladder cancer patient survival. In the right ceRNA network, 12 key lncRNAs (LINC00483, LPP-AS1, MIR205HG, WASIR2, OSBPL10-AS1, DSCAM-AS1, ZBTB20-AS1, ARHGEF38-IT1, C8 orf49, RMST, NOVA1-AS1, and KCNQ1OT1) were identified to be associated with clinical features. Among these 12 lncRNAs, LINC00483 was reported to regulate proliferation and apoptosis in gastric cancer (19); Di Agostino et al reported that MIR205HG led to unrestrained proliferation in head and neck squamous cell carcinoma by depleting miR-590-3p (27); DSCAM-AS1 could interact with miR-137 to enhance tamoxifen resistance in breast cancer (28). Microarray data revealed that lncRNA RMST was differentially expressed in cervical cancer, and the in vitro assay revealed that RMST played the role of tumour suppressor in TNBC by inhibiting cell proliferation, invasion and migration (29); some studies reported that KCNQ1OT1 played an important role in multiple malignancies by interacting with several miRNAs, such as miR-140-5p, miR-384b, miR-145, miR-211-5p, miR-7-5p and miR-504 (25,30-34). However, more research still needs to be carried out to confirm and understand the relationship between these RNAs and clinical features.

Our analysis has ramifications for the ceRNA network in colon cancer, and some results were confirmed by qRT-PCR. However, there are still several limitations in our study. First, the present study was derived only from the data of The Cancer Genome Atlas (TCGA) database, and the conclusion is relatively preliminary and requires validation from large-scale clinical trials. Second, our confirmation experiment is limited to qRT-PCR of two lncRNAs, 
Table IX. Expression of lncRNAs related to clinical features according to the clinicopathological characteristics of patients.

\begin{tabular}{|c|c|c|c|c|}
\hline \multirow[b]{2}{*}{ Characteristics } & \multirow[b]{2}{*}{ No. } & \multicolumn{2}{|c|}{ LINC00402 } & \multirow[b]{2}{*}{ P-value } \\
\hline & & Low group & High group & \\
\hline Age (years) & & & & 0.581 \\
\hline$<60$ & 20 & 11 & 9 & \\
\hline$\geq 60$ & 38 & 18 & 20 & \\
\hline Sex & & & & 0.597 \\
\hline Female & 26 & 12 & 14 & \\
\hline Male & 32 & 17 & 15 & \\
\hline Lymphatic metastasis & & & & 0.185 \\
\hline No & 33 & 14 & 19 & \\
\hline Yes & 25 & 15 & 10 & \\
\hline TNM staging system & & & & 0.03 \\
\hline $\mathrm{T} 1+\mathrm{T} 2$ & 22 & 7 & 15 & \\
\hline $\mathrm{T} 3+\mathrm{T} 4$ & 36 & 22 & 14 & \\
\hline Tumor stage & & & & 0.293 \\
\hline Stage I, II & 30 & 13 & 17 & \\
\hline Stage III, IV & 28 & 16 & 12 & \\
\hline MSI status & & & & 0.066 \\
\hline MSI-H & 14 & 5 & 9 & \\
\hline \multirow[t]{2}{*}{ Other status } & 44 & 22 & 12 & \\
\hline & & \multicolumn{2}{|c|}{ KCNQ1OT1 } & \\
\hline Characteristics & No. & Low group & High group & P-value \\
\hline Age (years) & & & & 0.279 \\
\hline$<60$ & 22 & 9 & 13 & \\
\hline$\geq 60$ & 36 & 20 & 16 & \\
\hline Sex & & & & 0.430 \\
\hline Female & 27 & 12 & 15 & \\
\hline Male & 31 & 17 & 14 & \\
\hline Lymphatic metastasis & & & & 0.007 \\
\hline No & 36 & 23 & 13 & \\
\hline Yes & 22 & 6 & 16 & \\
\hline TNM staging system & & & & 0.426 \\
\hline $\mathrm{T} 1+\mathrm{T} 2$ & 25 & 11 & 14 & \\
\hline $\mathrm{T} 3+\mathrm{T} 4$ & 33 & 18 & 15 & \\
\hline Tumour stage & & & & 0.033 \\
\hline Stage I, II & 34 & 21 & 13 & \\
\hline Stage III, IV & 24 & 8 & 16 & \\
\hline MSI status & & & & 0.557 \\
\hline MSI-H & 16 & 9 & 7 & \\
\hline Other status & 42 & 20 & 22 & \\
\hline
\end{tabular}

lncRNAs, long non-coding RNAs.

and further research is also required on the functions of key lncRNAs in vivo and in vitro, and the relationship of expression and function in the RNAs also requires further validation.
In conclusion, our research aimed to detect the difference between left and right colon cancer. By constructing ceRNA networks and analysing the relationship between the key lncRNAs and the clinical features, the putatively different 
mechanism of the two sides and the relationship among these 3 types of RNAs was partially revealed. The present study may further our insight into the difference between left and right colon cancer at the genetic level.

\section{Acknowledgements}

The authors would like to thank the faculty and staff at the Department of General Surgery (The First Affiliated Hospital of Nanjing Medical University, Jiangsu, China) for providing language and technology support.

\section{Funding}

The present study was funded by Jiangsu Key Medical Discipline-General Surgery (grant no. ZDXKA2016005).

\section{Availability of data and materials}

The data sets used and/or analysed during the present study are available from the corresponding author on reasonable request.

\section{Authors' contributions}

YS conceptualized and designed the research. WQ and YF performed the experiments, analysed and interpreted the results, made the figures and wrote the manuscript. JL, WP and QG performed the experiments and analysed the data. JL, ZZ and DJ provided the patient tissues. QG, ZZ and DJ also helped design the experimental studies and edited the manuscript. QW and DZ interpreted the results and wrote the manuscript. All authors read and approved the manuscript and agree to be accountable for all aspects of the research in ensuring that the accuracy or integrity of any part of the work are appropriately investigated and resolved.

\section{Ethics approval and consent to participate}

Thepresent study was approved by the ResearchEthicsCommittee of The First Affiliated Hospital of Nanjing Medical University, and written informed consent was obtained from all patients prior to enrolment in the study.

\section{Patient consent for publication}

Not applicable.

\section{Competing interests}

The authors declare that they have no competing interests.

\section{References}

1. Torre LA, Bray F, Siegel RL, Ferlay J, Lortet-Tieulent J and Jemal A: Global cancer statistics, 2012. CA Cancer J Clin 65 : 87-108, 2015.

2. Bufill JA: Colorectal cancer: Evidence for distinct genetic categories based on proximal or distal tumor location. Ann Intern Med 113: 779-788, 1990.

3. Gervaz P, Bucher P and Morel P: Two colons-two cancers: Paradigm shift and clinical implications. J Surg Oncol 88: 261-266, 2004.
4. Distler P and Holt PR: Are right- and left-sided colon neoplasms distinct tumors? Dig Dis 15: 302-311, 1997.

5. Glebov OK, Rodriguez LM, Nakahara K, Jenkins J, Cliatt J, Humbyrd CJ, DeNobile J, Soballe P, Simon R, Wright G, et al: Distinguishing right from left colon by the pattern of gene expression. Cancer Epidemiol Biomarkers Prev 12: 755-762, 2003.

6. Iacopetta B: Are there two sides to colorectal cancer? Int J Cancer 101: 403-408, 2002.

7. Sana J, Faltejskova P, Svoboda M and Slaby O: Novel classes of non-coding RNAs and cancer. J Transl Med 10: 103, 2012.

8. Pan W, Liu L, Wei J, Ge Y, Zhang J, Chen H, Zhou L, Yuan Q, Zhou C and Yang M: A functional lncRNA HOTAIR genetic variant contributes to gastric cancer susceptibility. Mol Carcinog 55: 90-96, 2016

9. Huang C, Cao L, Qiu L, Dai X, Ma L, Zhou Y, Li H, Gao M, Li W, Zhang Q, et al: Upregulation of H19 promotes invasion and induces epithelial-to-mesenchymal transition in esophageal cancer. Oncol Lett 10: 291-296, 2015.

10. Zheng HT, Shi DB, Wang YW, Li XX, Xu Y, Tripathi P, Gu WL, Cai GX and Cai SJ: High expression of lncRNA MALAT1 suggests a biomarker of poor prognosis in colorectal cancer. Int J Clin Exp Pathol 7: 3174-3181, 2014.

11. Salmena L, Poliseno L, Tay Y, Kats L and Pandolfi PP: A ceRNA hypothesis: The rosetta stone of a hidden RNA language? Cell 146: 353-358, 2011

12. Missiaglia E, Jacobs B, D'Ario G, Di Narzo AF, Soneson C, Budinska E, Popovici V, Vecchione L, Gerster S, Yan P, et al: Distal and proximal colon cancers differ in terms of molecular, pathological, and clinical features. Ann Oncol 25: 1995-2001, 2014.

13. Snaebjornsson P, Jonasson L, Jonsson T, Moller PH, Theodors A and Jonasson JG: Colon cancer in Iceland-a nationwide comparative study on various pathology parameters with respect to right and left tumor location and patients age. Int J Cancer 127: 2645-2653, 2010.

14. Livak KJ and Schmittgen TD: Analysis of relative gene expression data using real-time quantitative PCR and the $2^{-\Delta \Delta C \mathrm{~T}}$ method. Methods 25: 402-408, 2001.

15. Budinska E, Popovici V, Tejpar S, D'Ario G, Lapique N, Sikora KO, Di Narzo AF, Yan P, Hodgson JG, Weinrich S, et al: Gene expression patterns unveil a new level of molecular heterogeneity in colorectal cancer. J Pathol 231: 63-76, 2013.

16. Sadanandam A, Lyssiotis CA, Homicsko K, Collisson EA, Gibb WJ, Wullschleger S, Ostos LC, Lannon WA, Grotzinger C, Del Rio M, et al: A colorectal cancer classification system that associates cellular phenotype and responses to therapy. Nat Med 19: 619-625, 2013.

17. Zhang DY, Zou XJ, Cao CH, Zhang T, Lei L, Qi XL, Liu L and $\mathrm{Wu} \mathrm{DH}$ : Identification and functional characterization of long non-coding RNA MIR22HG as a tumor suppressor for hepatocellular carcinoma. Theranostics 8: 3751-3765, 2018.

18. Cui Z, An X, Li J, Liu Q and Liu W: LncRNA MIR22HG negatively regulates miR-141-3p to enhance DAPK1 expression and inhibits endometrial carcinoma cells proliferation. Biomed Pharmacother 104: 223-228, 2018.

19. Li D, Yang M, Liao A, Zeng B, Liu D, Yao Y, Hu G, Chen X, Feng Z, Du Y, et al: Linc00483 as ceRNA regulates proliferation and apoptosis through activating MAPKs in gastric cancer. J Cell Mol Med 15: 13661, 2018.

20. Lu PW, Li L, Wang F and Gu YT: Effects of long non-coding RNA HOST 2 on cell migration and invasion by regulating MicroRNA let-7b in breast cancer. J Cell Biochem 119: 4570-4580, 2018.

21. Macleod RJ: CaSR function in the intestine: Hormone secretion, electrolyte absorption and secretion, paracrine non-canonical Wnt signaling and colonic crypt cell proliferation. Best Pract Res Clin Endocrinol Metab 27: 385-402, 2013.

22. Basu S, Haase G and Ben-Ze'ev A: Wnt signaling in cancer stem cells and colon cancer metastasis. F1000Res 5: F1000, 2016.

23. Zarkou V, Galaras A, Giakountis A and Hatzis P: Crosstalk mechanisms between the WNT signaling pathway and long non-coding RNAs. Noncoding RNA Res 3: 42-53, 2018.

24. Lecarpentier Y, Claes V, Vallee A and Hébert JL: Interactions between PPAR gamma and the canonical wnt/beta-catenin pathway in type 2 diabetes and colon cancer. PPAR Res 2017: $5879090,2017$.

25. Feng W, Wang C, Liang C, Yang H, Chen D, Yu X, Zhao W, Geng D, Li S, Chen Z and Sun M: The dysregulated expression of KCNQ1OT1 and its interaction with downstream factors miR-145/CCNE2 in breast cancer cells. Cell Physiol Biochem 49: 432-446, 2018. 
26. Gao X, Ge J, Li W, Zhou W and Xu L: LncRNA KCNQ1OT1 ameliorates particle-induced osteolysis through inducing macrophage polarization by inhibiting miR-21a-5p. Biol Chem 399: 375-386, 2018

27. Di Agostino S, Valenti F, Sacconi A, Fontemaggi G, Pallocca M, Pulito C, Ganci F, Muti P, Strano S and Blandino G: Long non-coding MIR205HG depletes Hsa-miR-590-3p leading to unrestrained proliferation in head and neck squamous cell carcinoma. Theranostics 8: 1850-1868, 2018.

28. Ma Y, Bu D, Long J, Chai W and Dong J: LncRNA DSCAM-AS acts as a sponge of miR-137 to enhance tamoxifen resistance in breast cancer. J Cell Physiol 234: 2880-2894, 2019

29. Wang L, Liu D, Wu X, Zeng Y, Li L, Hou Y, Li W and Liu Z: Long non-coding RNA (LncRNA) RMST in triple-negative breast cancer (TNBC): Expression analysis and biological roles research. J Cell Physiol 233: 6603-6612, 2018.

30. Zhang X, Wang M, Sun H, Zhu T and Wang X: Downregulation of LINC00894-002 contributes to tamoxifen resistance by enhancing the TGF- $\beta$ signaling pathway. Biochemistry 83: 603-611, 2018.
31. Shen C, Kong B, Liu Y, Xiong L, Shuai W, Wang G, Quan D and Huang H: YY1-induced upregulation of lncRNA KCNQ1OT1 regulates angiotensin II-induced atrial fibrillation by modulating miR-384b/CACNA1C axis. Biochem Biophys Res Commun 505: 134-140, 2018.

32. Zhang S, Ma H, Zhang D, Xie S, Wang W, Li Q, Lin Z and Wang Y: LncRNA KCNQ1OT1 regulates proliferation and cisplatin resistance in tongue cancer via miR-211-5p mediated Ezrin/Fak/Src signaling. Cell Death Dis 9: 742, 2018.

33. Fan S, Fan C, Liu N, Huang K, Fang $X$ and Wang K: Downregulation of the long non-coding RNA ZFAS1 is associated with cell proliferation, migration and invasion in breast cancer. Mol Med Rep 17: 6405-6412, 2018

34. Li J, Wang W, Xia P, Wan L, Zhang L, Yu L, Wang L, Chen X, $\mathrm{Xiao} \mathrm{Y}$ and $\mathrm{Xu} \mathrm{C}$ : Identification of a five-lncRNA signature for predicting the risk of tumor recurrence in patients with breast cancer. Int J Cancer 143: 2150-2160, 2018. 